increased difficulty of achieving a greater percent reduction in the average daily dose when starting with a higher dose at baseline. No interaction was seen between the number of relapses during the previous 2 years and treatment effect. Overall, the results of the modeling were inconsistent among end points in terms of the strength of the evidence, and caution is needed in concluding any causal relationship between the factors that were evaluated and treatment effect.

Although approved only for eosinophilic asthma, ${ }^{1,2}$ mepolizumab has now shown efficacy in eosinophilic granulomatosis with polyangiitis and idiopathic hypereosinophilic syndrome. ${ }^{3}$ With its interleukin-5-blocking mechanism of action, it is likely that this therapy will have beneficial effects in other hypereosinophilic conditions, especially in intermediate phenotypes including the recently proposed entity of hypereosinophilic asthma with nonvasculitic systemic manifestations.

As with the patients in our study who did not have a response to mepolizumab, Kedar's case report of a patient who had a response to imatinib therapy after not having a response to other therapies, including reslizumab, highlights the need to do further studies to better understand the underlying pathobiologic mechanisms of eosinophilic granulomatosis with polyangiitis. Although the eosinophil appears to be the dominant cell implicated in most patients with eosinophilic granulomatosis with polyangiitis, it appears that this is a complex syndrome with several putative causative elements that make it difficult to differentiate from other hypereosinophilic syndromes. Given the apparent benefit of imatinib in Kedar's patient, we agree that future research exploring the role of tyrosine kinase inhibition in eosinophilic granulomatosis with polyangiitis is warranted.

Michael E. Wechsler, M.D.

National Jewish Health

Denver, CO

mikewechsler@gmail.com

Praveen Akuthota, M.D.

University of California, San Diego

La Jolla, CA

Paneez Khoury, M.D., M.H.S.

National Institutes of Health

Bethesda, MD

Since publication of their article, the authors report no further potential conflict of interest.

1. Ortega HG, Liu MC, Pavord ID, et al. Mepolizumab treatment in patients with severe eosinophilic asthma. $\mathrm{N}$ Engl J Med 2014;371:1198-207.

2. Pavord ID, Korn S, Howarth P, et al. Mepolizumab for severe eosinophilic asthma (DREAM): a multicentre, double-blind, placebo-controlled trial. Lancet 2012;380:651-9.

3. Rothenberg ME, Klion AD, Roufosse FE, et al. Treatment of patients with the hypereosinophilic syndrome with mepolizumab. N Engl J Med 2008;358:1215-28.

DOI: $10.1056 /$ NEJMc1708004

\title{
Exercise Type in Dieting Obese Older Adults
}

TO THE EDITOR: In a well-performed clinical trial, Villareal et al. (May 18 issue) ${ }^{1}$ found that weight loss plus physical activity improved functional status in obese older adults. Nevertheless, conclusions should be drawn with caution, because there was no weight-loss control group. We cannot differentiate whether the improvement in functional status was caused by weight loss, physical activity, or both. We are asked to assume that the observed increases in physical performance, peak oxygen consumption, and strength are attributable to increased physical activity, whereas the observed decreases in body weight, lean mass, and bone mineral density at the hip are attributable to the reduced-calorie diet. If these assumptions are correct, we would then have to conclude that it could be harmful to recommend weight loss by means of calorie restriction. In- deed, the "obesity paradox," observations of lower mortality among obese older adults than among older adults of normal weight, raises further questions about aiming for weight loss as an intervention of choice. We suggest a follow-up study with two comparison groups: one group consuming a calorie-restricted diet and another consuming a healthy diet (e.g., a Mediterranean $\operatorname{diet}^{3}$ or the DASH [Dietary Approaches to Stop Hypertension] $\operatorname{diet}^{4}$ ) that does not include calorie restriction.

Florian L. Stigler, M.D., M.P.H.

London School of Hygiene and Tropical Medicine

London, United Kingdom

florian.stigler@lshtm.ac.uk

Robert H. Lustig, M.D.

University of California, San Francisco

San Francisco, CA 
Adrian M. Moser, M.D.

Steiermärkische Gebietskrankenkasse

Graz, Austria

No potential conflict of interest relevant to this letter was reported.

1. Villareal DT, Aguirre L, Gurney AB, et al. Aerobic or resistance exercise, or both, in dieting obese older adults. N Engl J Med 2017;376:1943-55.

2. Dorner TE, Rieder A. Obesity paradox in elderly patients with cardiovascular diseases. Int J Cardiol 2012;155:56-65.

3. Estruch R, Ros E, Salas-Salvadó J, et al. Primary prevention of cardiovascular disease with a Mediterranean diet. N Engl J Med 2013;368:1279-90.

4. Steinberg D, Bennett GG, Svetkey L. The DASH diet, 20 years later. JAMA 2017;317:1529-30.

DOI: $10.1056 /$ NEJMc1708176

TO THE EDITOR: In their article on aerobic, resistance, and combined aerobic and resistance exercise modes in obese older adults, Villareal and colleagues conclude that the combination approach was the most effective in improving functional status. However, there are some points of discussion. Participants engaged in aerobic training and resistance training for approximately 180 minutes per week each, whereas the combination regimen resulted in approximately 270 minutes of exercise per week. We acknowledge that the volume of the combined aerobic and resistance regimen is in accordance with current guidelines,${ }^{1}$ but for a fair comparison, the volume should be equally distributed across training modes. We wonder whether the combined aerobic and resistance regimen would still show a higher benefit with respect to functional status than other training modes if the training volume was controlled. Indeed, a P value below the 0.05 threshold does not imply that the groups differed in the Physical Performance Test (PPT) scores. The between-group differences in the changes in the PPT score from baseline to 6 months were, in fact, small (albeit significant) (Table 2 of the article, available at NEJM.org). To determine whether the observed differences are clinically meaningful, it would be informative to provide mean differences of the changes in the PPT score (and the corresponding confidence intervals) among the groups at 3 months and 6 months. ${ }^{2}$

Leandro F.M. Rezende, M.Sc.

University of São Paulo

São Paulo, Brazil

lerezende@usp.br

Victor K.R. Matsudo, Ph.D.

Center of Studies of the Physical Fitness Research Laboratory

of São Caetano do Sul

São Paulo, Brazil
No potential conflict of interest relevant to this letter was reported.

1. American College of Sports Medicine Position Stand: the recommended quantity and quality of exercise for developing and maintaining cardiorespiratory and muscular fitness, and flexibility in healthy adults. Med Sci Sports Exerc 1998;30:97591.

2. Altman DG, Gore SM, Gardner MJ, et al. Statistical guidelines for contributors to medical journals. In: Altman D, Machin D, Bryant T, Gardner M, eds. Statistics with confidence. 2nd ed. London: BMJ Books, 2000:171-90.

DOI: 10.1056/NEJMc1708176

THE AUTHORS REPLY: The protocol for the present trial was informed by a previous study conducted by our group ${ }^{1}$ that included a weight-lossalone group as compared with non-weight-loss groups. Direct effects that were due to weight loss were established in that study. Differential effects were established in the present trial by the head-to-head comparison of aerobic exercise with resistance exercise during matched weight loss. Aerobic exercise resulted in greater improvements in cardiorespiratory fitness, and resistance exercise in greater improvements in strength; the combination exercise group had the greatest improvement in physical function. An important point of our present trial is that weight loss alone was harmful with respect to lean mass and bone mineral density but that the addition of resistance exercise or the combination of resistance and aerobic exercise attenuated the loss. Our ongoing study aims to determine whether an increase in bone strength compensates for bone loss that remains after resistance exercise or combined exercise is added to weight loss. The existence of an "obesity paradox" in older adults is unclear. ${ }^{2}$ Whether a Mediterranean diet or DASH diet would be as effective as a multifactorial intervention of weight loss and exercise in reversing frailty in obese older adults seems doubtful.

The differential outcomes that were due to the two exercise modes, aerobic and resistance, required that the combination exercise program ensured sufficient physiological responses from both exercise interventions. Therefore, the combination group did the full aerobic program plus the full resistance program in order to ensure an adequate dose of each type of training. Moreover, to test the interference effect, ${ }^{3}$ it was essential to balance the aerobic and resistance training between groups - reducing the training volume in the combination group would have made it impossible to determine whether a lesser physio- 
logical response was due to the lower volume or to the interference effect. Accordingly, the combination exercise program improved functional status the most, indicating no interference effect. We agree that a confidence interval of the difference includes more information than $P$ values in simple situations. Although, like P values, confidence intervals alone do not establish clinical importance, one needs some clinical idea of minimally important differences. ${ }^{4}$ The $95 \%$ confidence interval for the difference in the change in PPT scores between the combination group and either exercise group was 0.5 to 2.7 (in each comparison); the information needed to compute the confidence intervals between groups is provided in Table 2 of our article.

Dennis T. Villareal, M.D.

Baylor College of Medicine

Houston, TX

dennis.villareal@bcm.edu
Debra L. Waters, Ph.D.

University of Otago

Dunedin, New Zealand

Clifford Qualls, Ph.D.

University of New Mexico School of Medicine

Albuquerque, NM

Since publication of their article, the authors report no further potential conflict of interest.

1. Villareal DT, Chode S, Parimi N, et al. Weight loss, exercise, or both and physical function in obese older adults. $\mathrm{N}$ Engl $\mathrm{J}$ Med 2011;364:1218-29.

2. Bowman K, Delgado J, Henley WE, et al. Obesity in older people with and without conditions associated with weight loss: follow-up of 955,000 primary care patients. J Gerontol A Biol Sci Med Sci 2017;72:203-9.

3. Wilson JM, Marin PJ, Rhea MR, Wilson SM, Loenneke JP, Anderson JC. Concurrent training: a meta-analysis examining interference of aerobic and resistance exercises. J Strength Cond Res 2012;26:2293-307.

4. Revicki D, Hays RD, Cella D, Sloan J. Recommended methods for determining responsiveness and minimally important differences for patient-reported outcomes. J Clin Epidemiol 2008;61:102-9.

DOI: 10.1056/NEJMc1708176

\section{Case 14-2017: A Man with Pain and Swelling of the Left Calf and a Purpuric Rash}

TO THE EDITOR: In the discussion of Case 142017 (May 11 issue), ${ }^{1}$ a patient who had Crohn's disease and was receiving adalimumab therapy presented with IgA vasculitis. An underlying cancer perhaps should have been considered, despite the absence of "constitutional symptoms, lymphadenopathy, or a testicular mass." In adults, an association between IgA vasculitis and cancer especially pulmonary, prostatic, and renal carcinomas but also hematologic cancer - has been described. ${ }^{2,3}$ Adalimumab therapy has also been associated with cancer; for example, in a prospective registry, the risk of lymphoma was two to three times as high among patients who were receiving adalimumab as it was among those who were not. ${ }^{4}$ However, a meta-analysis has suggested that anti-tumor-necrosis-factor (TNF) therapy is not associated with an increased risk of cancer in patients with inflammatory bowel diseases, although there may have been an increase in skin cancer and lymphoma among those patients. ${ }^{5}$ The data in the meta-analysis are from cohort studies, postmarketing registries, and meta- analyses of randomized trials, all with methodologic weaknesses. ${ }^{5}$

François Rodrigues, M.D.

Sophie Georgin-Lavialle, M.D., Ph.D.

Claude Bachmeyer, M.D.

Hôpital Tenon

Paris, France

claude.bachmeyer@tnn.aphp.fr

No potential conflict of interest relevant to this letter was reported.

1. Case Records of the Massachusetts General Hospital (Case 14-2017). N Engl J Med 2017;376:1868-77.

2. Pertuiset E, Lioté F, Launay-Russ E, Kemiche F, CerfPayrastre I, Chesneau AM. Adult Henoch-Schönlein purpura associated with malignancy. Semin Arthritis Rheum 2000;29:360-7. 3. Podjasek JO, Wetter DA, Pittelkow MR, Wada DA. HenochSchönlein purpura associated with solid-organ malignancies: three case reports and a literature review. Acta Derm Venereol 2012;92:388-92.

4. Mariette X, Tubach F, Bagheri H, et al. Lymphoma in patients treated with anti-TNF: results of the 3-year prospective French RATIO registry. Ann Rheum Dis 2010;69:400-8.

5. Williams CJ, Peyrin-Biroulet L, Ford AC. Systematic review with meta-analysis: malignancies with anti-tumour necrosis factor- $\alpha$ therapy in inflammatory bowel disease. Aliment Pharmacol Ther 2014;39:447-58.

DOI: $10.1056 /$ NEJMc1707419 